\title{
Expression of laminin in benign and malignant sclerosing lesions of extrahepatic bile ducts
}

\author{
C HAGLUND, P J ROBERTS, S NORDLING* \\ From the Fourth Department of Surgery, Helsinki University Central Hospital, and the *Department of \\ Pathology, University of Helsinki, Helsinki, Finland
}

SUMMARY The distribution of laminin, a basement membrane glycoprotein, was studied by an immunohistochemical technique in six samples of benign sclerosing lesions of extrahepatic bile ducts and in 11 sclerosing cholangiocarcinomas. The expression of laminin showed that benign glandular structures were surrounded by a mainly intact basement membrane. In sclerosing cholangiocarcinomas laminin was irregularly distributed, and in large areas totally absent. The findings suggest that deposition of basement membrane material is defective. Sclerosing cholangitis may be extremely difficult to distinguish from well differentiated sclerosing cholangiocarcinoma. This sample was small, but the diseases studied were relatively rare.

Staining for laminin might be of additional use to surgical pathologists in the differential diagnosis of benign sclerosing lesions and sclerosing cholangiocarcinomas.

Disorders of the extrahepatic bile duct, which cause fibrous thickening of the wall and stenosis of the lumen, may be reactive to an inflammatory condition, or may represent a malignant disease. The condition presents a difficult diagnostic problem both to surgeons and surgical pathologists. Benign bile duct strictures may be due to primary sclerosing cholangitis, a rare condition of unknown aetiology, characterised by chronic fibrosis and inflammation, or to so called "secondary" sclerosing cholangitis, which may follow on from common bile duct stones or previous biliary surgery. In these conditions atypical glands are often present in the wall and may therefore be confused with well differentiated sclerosing cholangiocarcinoma, a variant of bile duct carcinoma. ${ }^{1-4}$

Several studies have shown that loss of basement membrane integrity is characteristic of different types of malignant epithelial cells. ${ }^{5-13}$ Laminin is a large glycoprotein which is synthesised by many types of cells and is present in both epithelial and vascular basement membranes. ${ }^{14} 15$

In this study we evaluated whether demonstration of basement membranes using laminin antibodies

Accepted for publication 20 April 1989 might help to distinguish between benign and malignant sclerosing lesions of extrahepatic bile ducts.

\section{Material and methods}

Specimens obtained from extrahepatic sclerosing lesions were as follows: two of primary sclerosing cholangitis; four of secondary sclerosing cholangitis (two cases of postoperative fibrosis and two strictures following common bile duct stones); and 11 of sclerosing cholangiocarcinoma.

Routinely processed paraffin wax blocks of formaldehyde fixed tissues had been stored for one to 10 years. Dewaxed tissue sections were reacted with rabbit antibodies against mouse laminin, which cross-reacts with human laminin, ${ }^{1617}$ and bound antibody was visualised by the avidin-biotin complex technique (ABC) (Vector Laboratories, Burlingame, California, USA), as described previously. ${ }^{11}$ No nuclear counterstain for nuclei was used. Perivascular laminin staining was seen in all sections, which served as an internal positive control. ${ }^{18}$ Treatment with pepsin made it possible to detect laminin in formalin fixed tissues. ${ }^{18}$ The optimal dilution of the primary antibody was determined in a control series. Control staining with pre-immune serum was negative in all cases. Conventional staining techniques were used for routine histological examination. 


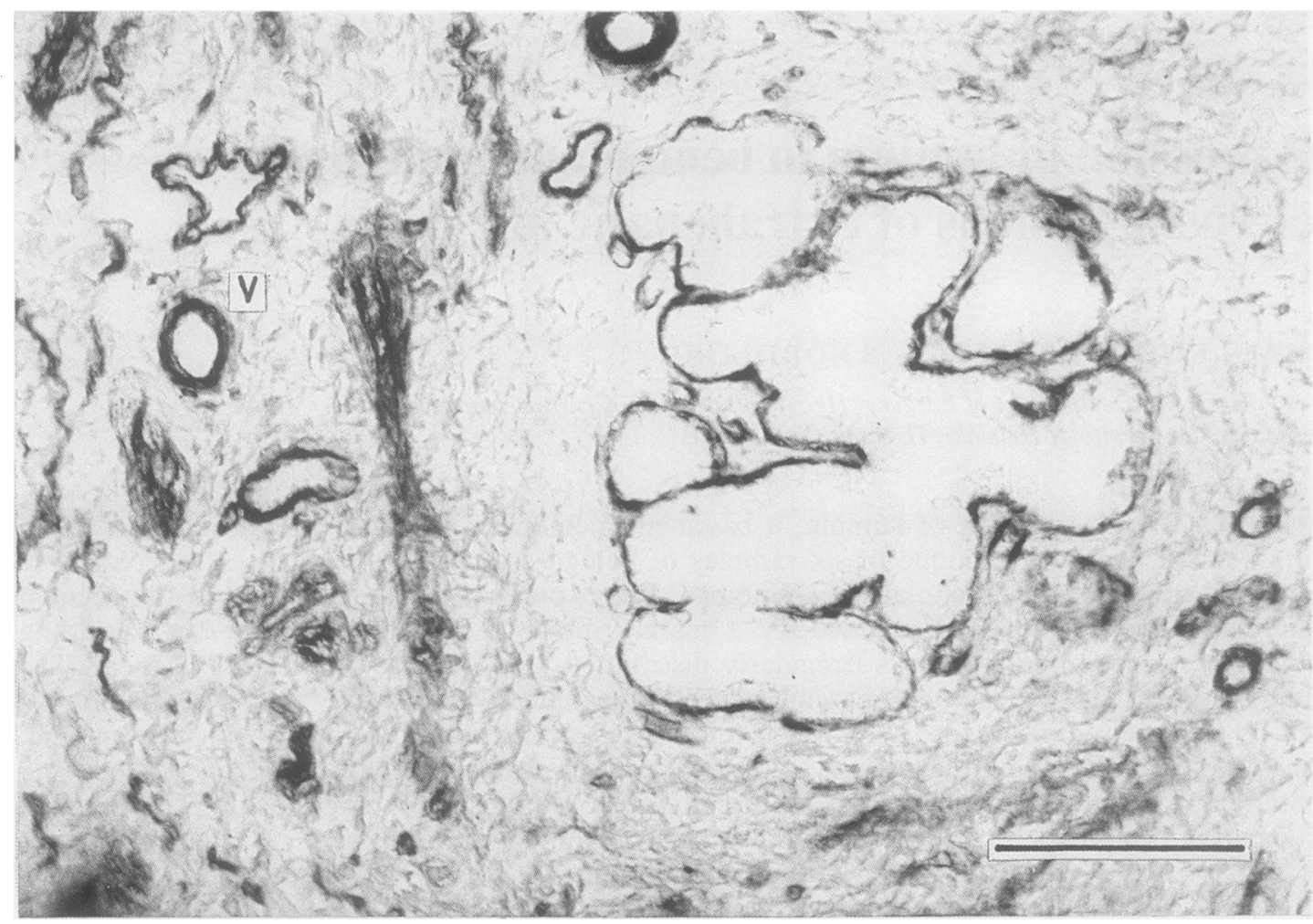

Fig 1 Sclerosing cholangitis. Glandutar structures are surrounded by a mainly intact layer of laminin. Blood vessels are positive for laminin (V). (Immunoperoxidase staining with laminin antibodies, bar $=100 \mu \mathrm{m}$ ).

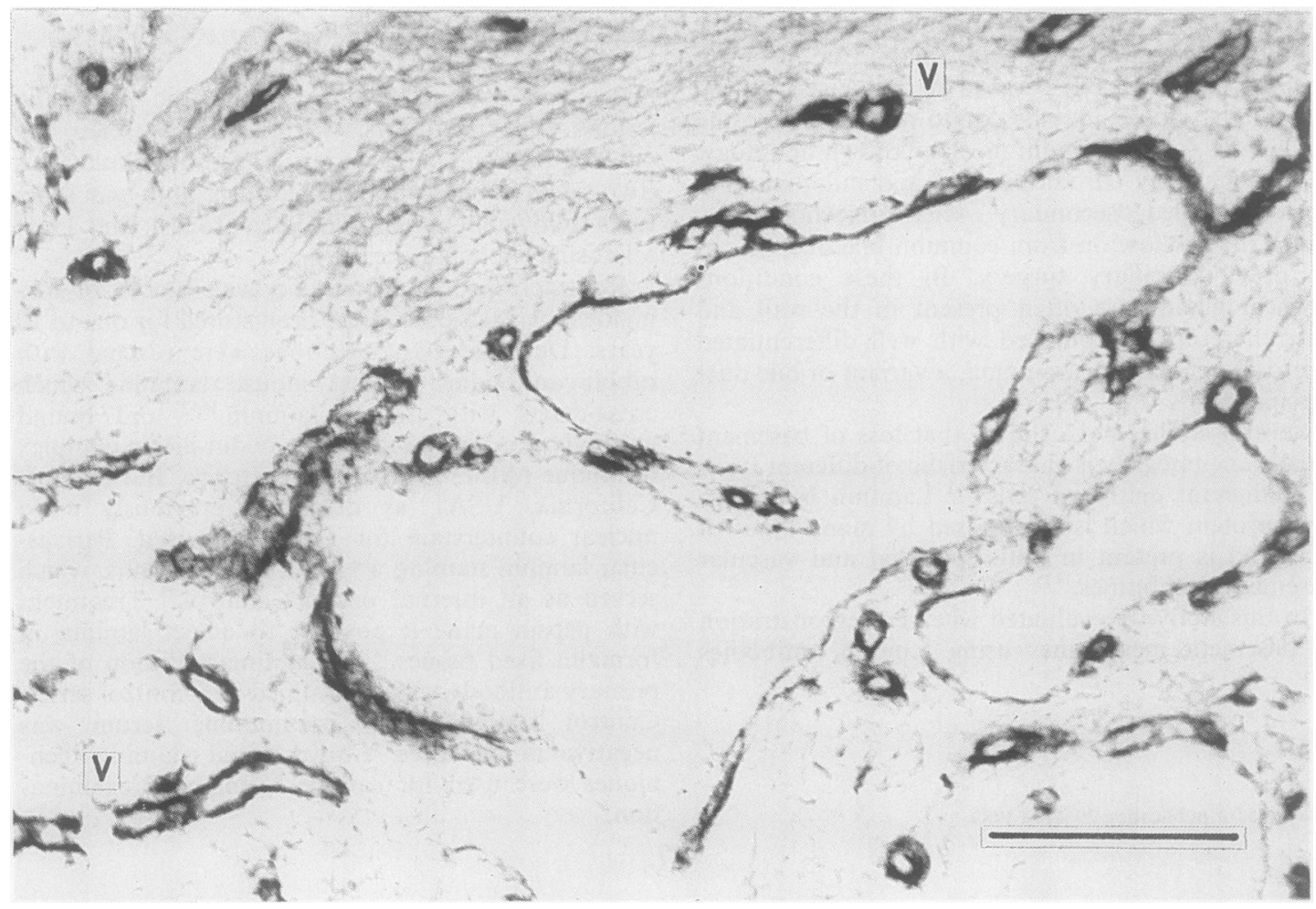

Fig 2 Well differentiated sclerosing cholangiocarcinoma. Malignant glands are surrounded by an irregular and discontinuous basement membrane. (Immunoperoxidase staining with laminin antibodies, bar $=100 \mu \mathrm{m}, \mathrm{V}=$ blood vessels.) 


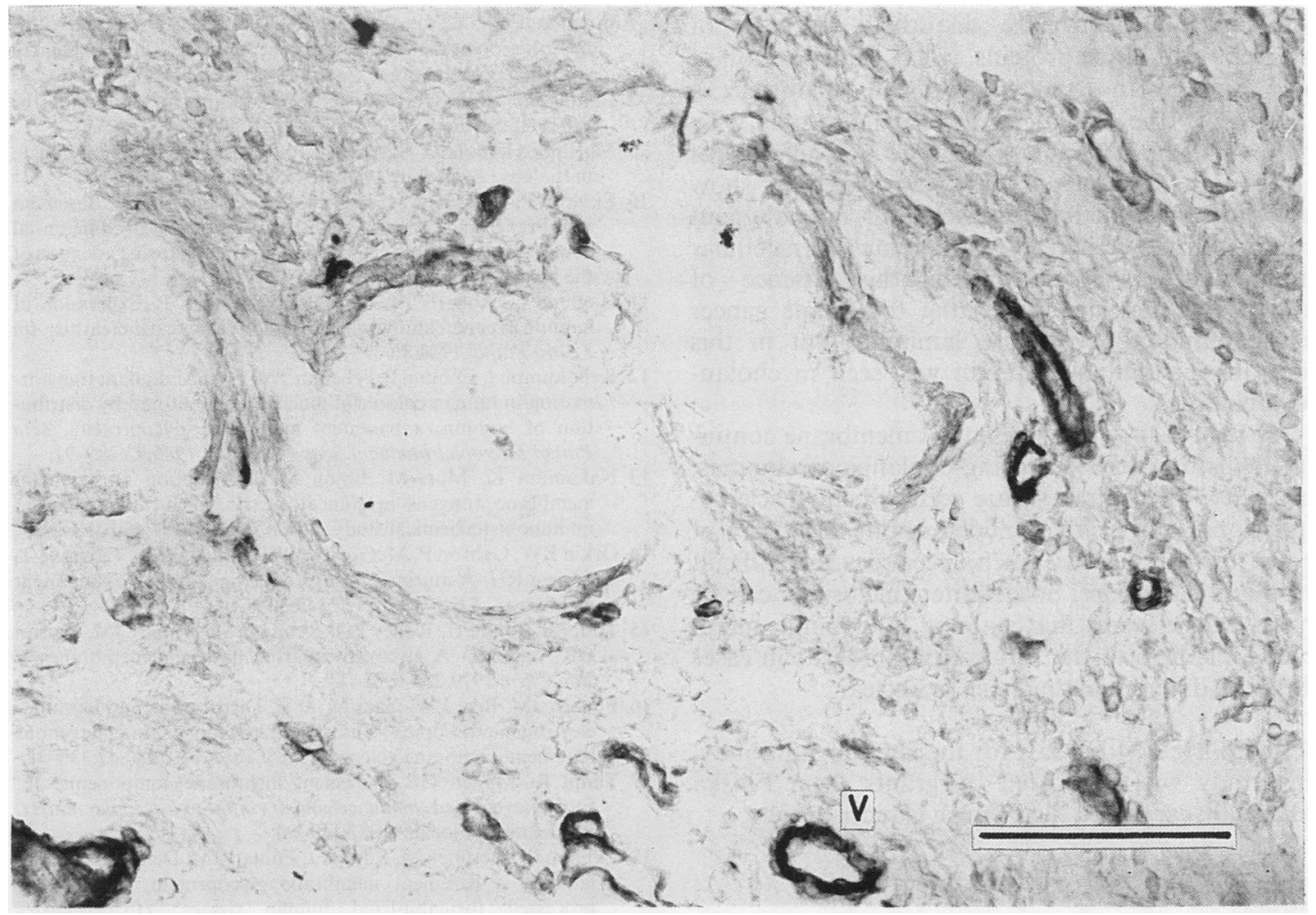

Fig 3 Well differentiated sclerosing cholangiocarcinoma. In this specimen only patches of laminin was seen around glandular structures. (Immunoperoxidase staining with laminin antibodies, bar $=100 \mu \mathrm{m}, \mathrm{V}=$ blood vessels.)

\section{Results}

In benign sclerosing lesions, both in primary sclerosing cholangitis and in secondary fibrosing lesions, glandular structures were surrounded by a mainly intact layer of laminin (fig 1). Only a few short breaks in continuity were seen. Nerves and vessel walls were positive for laminin. No positive staining was seen inside the cells.

In sclerosing cholangiocarcinomas there was an almost intact basement membrane around some well differentiated glands, but around most glands the basement membrane was irregular and discontinuous (fig 2). In places, even in well differentiated areas, only patches of laminin were seen (fig 3). In moderately differentiated areas the basement membrane was always clearly affected and in poorly differentiated areas only patches of laminin were seen, or laminin was totally absent. No intracellular staining was seen.

\section{Discussion}

The clinicopathological features of benign and malig- nant disorders affecting the extrahepatic bile ducts may be similar. Anamnestic data may help to distinguish postoperative stenosis from other conditions, and endoscopic retrograde cholangiography (ERCP) or percutaneous cholangiography (PTC) may lead to a correct diagnosis, but in many cases laparotomy is needed. Benign sclerotic processes and sclerosing cholangiocarcinoma may be difficult to distinguish even from adequate biopsy specimens taken during laparotomy. Biopsy specimens are often small. Sclerosing cholangiocarcinomas are often extremely well differentiated. In benign disorders atypical intramural duct-like structures, involved in the sclerosing process, may histologically mimic neoplastic glands. Because the choice of treatment depends on the histopathological diagnosis, it is important to find methods which distinguish between these conditions. ${ }^{1-4}$

Malignant transformation is accompanied by the loss of an intact basement membrane. This has been shown in different carcinomas using antisera against various basement membrane components. ${ }^{5-1316}$ This study shows similar results for sclerosing cholangiocarcinomas. Possible mechanisms of basement 
membrane deficiency are disturbed synthesis of basement membrane proteins, a lack of deposition of these proteins to form a basement membrane, a masking of the basement membrane proteins by unknown deposits, and proteolytic degradation of formed basement membranes. Several reports show that proteolytic activity is increased in malignant tumours. ${ }^{19-21}$ In pancreatic carcinomas intracellular laminin has been shown in the absence of extracellular deposits, suggesting that some cancer cells continue to synthesise laminin, " but in this study no intracellular laminin was seen in cholangiocarcinomas.

Our results show that basement membrane continuity is disturbed in sclerosing cholangiocarcinomas; only a few discontinuities are seen in benign sclerosing lesions. This study included only a few cases of benign lesions, because such specimens are difficult to obtain. Therefore, no definite conclusions can be drawn, but it seems that staining for laminin might be of some help to the surgical pathologists in cases where the differential diagnosis is unclear.

We thank Dr J M Foidart for the lamining antibody. This study was supported by grants from Finska Läkaresällskapet and the Finnish Cancer Society.

\section{References}

1 Chapman RWG, Arborgh BÅM, Rhodes JM, et al. Primary sclerosing cholangitis: a review of its clinical features, cholangiography, and hepatic histology. Gut 1980;21:870-7.

2 Williams LF Jr, Schoetz JD Jr. Primary sclerosing cholangitis. Surg Clin North Am 1981;61:951-62.

3 Lefkowitch JH. Primary sclerosing cholangitis. Arch Intern Med 1982;142:1157-60.

4 Weinbren K, Mutum SS. Pathological aspects of cholangiocarcinoma. J Pathol 1983;139:217-38.

5 Albrechtsen R, Nielsen M, Wewer U, Engvall E, Ruoslahti E. Basement membrane changes in breast cancer detected by immunochemical staining for laminin. Cancer Res 1981;41:5076-81.

6 Burtin P, Chavanel G, Foidart JM, Martin E. Antigens of the basement membrane and the peritumoral stroma in human colonic adenocarcinomas: An immunofluorescence study. Int J Cancer 1982;30:13-20.
7 Birembaut $\mathrm{Ph}$, Caron $\mathrm{Y}$, van Cauwen-Berge D, Foidart JM Distribution of laminin, a basement membrane glycoprotein in epithelial proliferations. Collat Rel Res 1983;3:25-31.

8 Liotta LA, Rao CN, Barsky SH. Tumor invasion and the extracellular matrix. Lab Invest 1983;49:636-49.

9 Martinez-Hernandez A, Amenta PS. The basement membrane in pathology. Lab Invest 1983;48:656-77.

10 Ekblom P, Miettinen M, Forsman L, Andersson LC. Basement membrane and apocrine epithelial antigens in differential diagnosis between tubular carcinoma and sclerosing adenosis of the breast. J Clin Pathol 1984;37:357-63.

11 Haglund C, Roberts PJ, Nordling S, Ekblom P. Expression of laminin in pancreatic neoplasms and in chronic pancreatitis. Am J Surg Pathol 1984;8:669-76.

12 Kellokumpu I, Ekblom P, Scheinin TM, et al. Malignant transformation in human colorectal mucosa as monitored by distribution of laminin, a basement membrane glycoprotein. Acta Pathol Microbiol Immunol Scand (Sect A) 1985;93:285-91.

13 Nakamura K, Mori M, Enjoji M. Distribution of basement membrane antigens in clinical gastric adenocarcinomas: an immunohistochemical study. J Clin Pathol 1987;40:1418-23.

14 Orkin RW, Gehron P, McGoodwin EB, Martin GR, Valentine T, Swarm RH. A murine tumor producing a matrix of basement membrane. J Exp Med 1977;145:204-20.

15 Timpl R, Rohde H, Robey PG, Rennard SI, Foidart JM, Martin GR. Laminin- A glycoprotein from basement membranes. $J$ Biol Chem 1979;254:9933-7.

16 Foidart JM, Bere EW, Yaar M, et al. Distribution and immunoelectron microscopic localisation of laminin, a non-collagenous basement membrane glycoprotein. Lab Invest 1980;42:336-42.

17 Timpl R, Martin GR. Basement membrane components. In: Furthmeyer $\mathrm{H}$, ed. Immunochemistry of the extracellular matrix. Boca Raton, Florida: 1982:119-50.

18 Ekblom P, Miettinen M, Rapola J, Foidart JM. Demonstration of laminin, a basement membrane glycoprotein, in routinely processed formalin-fixed human tissues. Histochemistry 1982;75:301-7.

19 Recklies AD, Tiltman KJ, Stoker AM, Poole AR. Secretion of proteinases from malignant and non-malignant human breast tissue. Cancer Res 1980;40:550-6.

20 Vetterlein D, Bell TE, Young PL, Roblin R. Immunological quantitation and immunoadsorption of urokinase-like plaminogen activators secreted by human cells. $J$ Biol Chem 1980;255:3665-72.

21 Liotta LA, Goldfarb RH, Brundage R, Siegal GP, Terranova VP, Garbisa S. Effect of plasminogen activator (urokinase), plasmin, and thrombin on glycoprotein and collagenous components of basement membrane. Cancer Res 1981;41:4629-36.

Requests for reprints to: Dr Caj Haglund, Fourth Department of Surgery, Helsinki University Central Hospital, Kasarmikatu 11-13, SF-00130 Helsinki, Finland. 